Trauma Berufskrankh 2015 • [Suppl 2]:

17:322-329

DOI 10.1007/s10039-015-0026-4

Online publiziert: 21. Mai 2015

๑) Springer-Verlag Berlin Heidelberg 2015

\author{
Axel Kramer ${ }^{1}$ - Claus-Dieter Heidecke ${ }^{2,3}$ \\ ${ }^{1}$ Institut für Hygiene und Umweltmedizin, Universitätsmedizin Greifswald, Greifswald, Deutschland \\ 2 Klinik für Chirurgie, Abt. für Allgemeine, Viszeral-, Thorax- und Gefäßchirurgie, \\ Universitätsmedizin Greifswald, Greifswald, Deutschland \\ ${ }^{3}$ Chirurgische Arbeitsgemeinschaft für Qualität und Sicherheit in der Chirurgie \\ (CAQS), Deutsche Gesellschaft für Chirurgie, Berlin, Deutschland
}

\title{
Präoperative Hautantiseptik und Hautschutz
}

fektionen und beatmungsassoziierter Pneumonien.

[68] lagen postoperative Wundinfektionen („surgical site infections“, SSI) in deutschen Akutkrankenhäusern mit ca. $16 \%$ an 3. Stelle der nosokomialen Infektionen nach nosokomialen Pneumonien und nosokomialen Harnwegsinfektionen. Im Vergleich dazu sind sie, wie die Prävalenzerhebung des European Centre for Disease Prevention and Control [61] ergab, $2011 \mathrm{mit} 24,7 \%$ an die erste Stelle der nosokomialen Infektionen gerückt, gefolgt von Harnwegsinfektionen mit 22,4\% und unteren Atemwegsinfektionen mit 21,5\%. Der Anteil von Infektionen mit Clostridium difficile betrug $6,6 \%$, der von primärer Sepsis $6,0 \%$. Als häufigste Erreger nosokomialer Infektionen wurden Escherichia coli (18,4\%), Staphylococcus (S.) aureus (13,3\%) und Enterococcus faecalis sowie Enterococcus faecium (12,8\%) beobachtet [61]. Aufgrund seiner vielfältigen Virulenzfaktoren ist $S$. aureus in akut traumatischen, chirurgischen, chronischen und Verbrennungswunden als der problematischste Erreger anzusehen.

Für die Verschiebung der SSI auf Platz 1 kommen u. a. folgende Ursachen in Betracht:

- das immer höhere Alter operativ zu versorgender Patienten,

- die Durchführung bisher nicht möglich gewesener Operationen aufgrund neuer Operationstechniken,

- die Zunahme multiresistenter Erreger und

- die im Vergleich zur Prävention von SSI einfacher realisierbare Prävention katheterassoziierter Harnwegsin-
Unabhängig davon müssen im Sinne der Patientensicherheit alle Möglichkeiten ausgeschöpft werden, die gesicherten Maßnahmen zur Prävention von SSI als Multibarrierenstrategie im Zusammenwirken aller Teammitglieder umzusetzen [53]. Dies ist nicht nur eine ethische Herausforderung, sondern auch unter ökonomischen Gesichtspunkten unerlässlich. Fall-Kontroll-Studien haben gezeigt, dass für Patienten mit SSI die Wahrscheinlichkeit zu sterben 2-fach höher, auf der Intensivstation behandelt zu werden, $60 \%$ und neuerlich im Krankenhaus behandelt zu werden, 5-fach höher ist. Durchschnittlich entstehen durch eine SSI Kosten von ca. 3000 EUR und die Aufenthaltsdauer in der Klinik verlängert sich um ca. 6,5 Tage [36]. Hochgerechnet bedeutet dies eine Belastung für die Versicherungssysteme von fast 300 Mio. EUR Mehrkosten und 614.000 zusätzliche Krankenhausbehandlungstage pro Jahr in Deutschland [52].

\section{Präoperative Hautantiseptik}

\section{Evidenz}

Die präoperative Hautantiseptik nimmt eine Schlüsselstellung in der Prävention von SSI ein, da bei der Durchtrennung der Haut eine Verschleppung der residenten Hautflora in die Tiefe des Operationsfelds verhindert werden soll. Die durchschnittliche Dichte der residenten Mikroflora schwankt zwischen $10^{2}$ und $10^{6} \mathrm{KBE} / \mathrm{cm}^{2}$ Haut. Dabei sind hohe Be- siedlungsdichten auf talgdrüsenreichen, feuchten Arealen wie Rücken und Sternum $\left(10^{3}-10^{5} \mathrm{KBE} / \mathrm{cm}^{2}\right)$, Stirn, Kopfhaut und Axilla $\left(10^{4}-10^{6} \mathrm{KBE} / \mathrm{cm}^{2}\right) \mathrm{zu}$ finden. Auf trockenen, talgdrüsenarmen Arealen sind die Besiedlungsdichten üblicherweise geringer, z. B. auf Händen, Armen oder Beinen ca. $10^{2}-10^{3} \mathrm{KBE} / \mathrm{cm}^{2}$, auf der Fußsohle $10^{2}-10^{4} \mathrm{KBE} / \mathrm{cm}^{2}$ und auf dem $\mathrm{Ab}$ domen $10^{3}-10^{4} \mathrm{KBE} / \mathrm{cm}^{2}$ [26]. Die residente Flora besiedelt überwiegend das Stratum corneum und die distalen Abschnitte von Haarfollikeln und Talgdrüsenausführungsgängen; etwa ein Fünftel ist in einer Tiefe von $>0,3 \mathrm{~mm}$ zu finden. Sie enthält vorwiegend S. epidermidis (in $25 \%$ aller Isolate nachweisbar), weitere koagulasenegative Staphylokokken, koryneforme Stäbchenbakterien wie Propionibacterium spp., Corynebacterium spp., Dermabacteria spp. und Microccocus spp., ferner Pityrosporum spp. Für die temporär residente Flora hat insbesondere $S$. aureus Bedeutung, gefolgt von Acinetobacter spp. und Dermatophyten [27, 35].

Die Bedeutung der Hautflora als Quelle für SSI wird durch folgende Zusammenhänge untermauert:

- In Abhängigkeit von der Formulierung eingesetzter Hautantiseptika resultiert eine unterschiedliche SSI-Rate (s. u.).

- Durch Rasur im Operationsfeld wird in Abhängigkeit vom Abstand zur Operation das SSI-Risiko signifikant erhöht, sodass die Rasur obsolet ist und nur im Fall einer erforderlichen Haarentfernung Clipping anstelle Rasur durchgeführt werden soll [40]. 


\begin{tabular}{|c|c|c|c|c|}
\hline Antiseptische Formulierung & Studientyp & Eingriff & Ergebnis & Quelle \\
\hline $\begin{array}{l}0,5 \% \text { Chx-Alkohol (A) vs. PVP-lod (\%?) in } \\
\text { Alkohol (B) }\end{array}$ & $\begin{array}{l}\text { Randomisiert, Verblindung } \\
\text { unklar, Alkohol nicht ange- } \\
\text { geben (vermutlich Ethanol) }\end{array}$ & Aseptisch & A signifikant wirksamer & Berry et al. [9] \\
\hline $2 \%$ lod in $90 \%$ Eth vs. $70 \%$ Eth (je $1 \mathrm{~min})$ & Randomisiert & Aseptisch & $\begin{array}{l}\text { Kein Unterschied (unklares } \\
\text { Biasrisiko) }\end{array}$ & Alexander et al. [2] \\
\hline $\begin{array}{l}10 \% \text { PVP-lod (A) vs. } 0,5 \% \text { Chx (B), je in } \\
70 \% \text { Eth }\end{array}$ & Randomisiert, prospektiv & $\begin{array}{l}\text { Elektive plastische } \\
\text { Chirurgie }\end{array}$ & $\begin{array}{l}\text { B tendenziell wirksamer } \\
(p=0,06)\end{array}$ & Veiga et al. [82] \\
\hline $\begin{array}{l}\text { Wässrige PVP-lod-Lösung (A) vs. lodpo- } \\
\text { vacrylex in } 70 \% \text { iProp (B) vs. } 2 \% \text { Chx in } \\
70 \% \text { iProp (C) }\end{array}$ & Sequenziell & & $\begin{array}{l}\text { B signifikant wirksamer als } \\
\text { A und C, in Subgruppen- } \\
\text { analyse unterschied sich A } \\
\text { nicht von B }\end{array}$ & Swenson et al. [73] \\
\hline $\begin{array}{l}7,5 \% \text { PVP-Waschung, danach } 10 \% \text { wäss- } \\
\text { riges PVP-lod vs. } 4 \% \text { Chx-Waschung, } \\
\text { danach } 4 \% \mathrm{Chx}\end{array}$ & Prospektiv randomisiert & $\begin{array}{l}\text { Aseptisch, bedingt } \\
\text { aseptisch, septisch }\end{array}$ & Kein Unterschied & Paocharoen et al. [63] \\
\hline $\begin{array}{l}\text { Wässrige PVP-lod-Lösung (A) vs. } 2 \% \text { Chx } \\
\text { in } 70 \% \text { iProp (B) }\end{array}$ & Randomisiert & $\begin{array}{l}\text { Sauber kontaminiert, } \\
\text { Viszeralchirurgie }\end{array}$ & B signifikant wirksamer & Darouiche et al. [12] \\
\hline $\begin{array}{l}\text { Wässrige } 10 \% \text { PVP-lod Lösung (A) vs. } 2 \% \\
\text { Chx in } 70 \% \text { Eth (B) }\end{array}$ & Prospektiv randomisiert & $\begin{array}{l}\text { Aseptisch, Inguinal- } \\
\text { hernie }\end{array}$ & B tendenziell wirksamer & Sistla et al. [70] \\
\hline $\begin{array}{l}10 \% \text { PVP-lod-Waschung, danach } 10 \% \\
\text { PVP-lod in } 65 \% \text { Eth (A) vs. Waschung mit } \\
\text { Chx } 2 \% \text {, danach } 70 \% \text { Eth (B) }\end{array}$ & Retrospektiv & $\begin{array}{l}\text { Elektive gynäkologi- } \\
\text { sche Laparotomie }\end{array}$ & B signifikant wirksamer & Levin et al. [50] \\
\hline $\begin{array}{l}\text { Wässrige PVP-lod-Lösung (A) vs. } 2 \% \text { Chx } \\
\text { in } 70 \% \text { iProp (B) }\end{array}$ & $\begin{array}{l}\text { Retrospektive Kohorten- } \\
\text { studie }\end{array}$ & Kaiserschnitt & Kein Unterschied & Menderes et al. [58] \\
\hline $\begin{array}{l}\text { PVP-lod } 10 \% \text { Waschung, danach } 10 \% \\
\text { PVP-lod in } 65 \% \text { Eth (A) vs. Waschung mit } \\
\text { Chx } 2 \% \text {, danach } 70 \% \text { Eth }\end{array}$ & Retrospektiv & Kaiserschnitt & B signifikant wirksamer & Amer-Alshiek et al. [3] \\
\hline
\end{tabular}

- Durch Anwendung nicht antiseptisch imprägnierter Inzisionsfolie wird das SSI-Risiko signifikant erhöht [84].

- Dagegen kann durch präoperative Hautversiegelung das SSI-Risiko reduziert werden, wenn auch aufgrund der inkonsistenten Datenlage derzeit dazu keine Empfehlung abgeleitet werden kann [15, 17, 34, 76, 83, 86].

Als Ursache für die Bedeutung der Hautantiseptik ist anzusehen, dass insbesondere aus den tieferen Schichten der Haut einschließlich der Talg- und Schweißdrüsen in die Operationswunde gelangende Erreger eine Infektion verursachen können, da die Mikroflora der Haut durch die Hautantiseptik nicht komplett eliminierbar ist [80]. Daher ist die präoperative Hautantiseptik in der Empfehlung der Kommission für Krankenhaushygiene und Infektionsprävention (KRINKO) zur Prävention postoperativer Infektionen im Operationsgebiet [42] mit der Evidenzkategorie IB gemäß Kategorisierung der KRINKO [44] eingeordnet. Nur folgende Maßnahmen zur Prävention von SSI er- füllen die Anforderungen an die Evidenzkategorie IA [43, 53]:

- Sanierung bestehender Infektionen vor elektiven Eingriffen

- Qualitätsgerechte Aufbereitung von Medizinprodukten

- Indikationsgerechte perioperative Antibiotikaprophylaxe

- Clipping oder keine Rasur

- Vermeidung akzidenteller Hypothermie

- Surveillance von SSI

\section{Wirkstoffbasis und Wirksamkeit}

Aufgrund ihrer raschen Wirkung und hohen Wirksamkeit sind alkoholbasierte Formulierungen für die Hautantiseptik Wirkstoff der ersten Wahl [16]. Im Praxistest auf talgdrüsenarmer Haut wird für die mit alkoholischen Präparaten innerhalb von 15 s erreichte Wirksamkeit mit wässriger Povidon(PVP)-Iod-Lösung 1 min benötigt. Auf talgdrüsenreicher Haut ist der Unterschied mit 2,5 min (für einige alkoholbasierte Formulierungen) vs. 10 min für wässrige PVP-Iod-Lösung noch ausgeprägter (Desinfektionsmittel- liste des Verbunds für Angewandte Hygiene, VAH, [81]). Analog ist die Wirksamkeit wässriger Lösungen auf Basis von PVP-Iod oder Chlorhexidindigluconat $(\mathrm{Chx})$ zur hygienischen Händedesinfektion deutlich geringer als die von Alkoholen [67]. Noch größer ist die Wirkungsdifferenz zwischen wässriger Lösung auf Basis von PVP-Iod oder Chx und Alkoholen auf die residente Flora im Rahmen der chirurgischen Händedesinfektion [66].

Während bei dermaler Injektion und Punktion keine remanente Wirksamkeit des Hautantiseptikums erforderlich ist, da die Wirkung nur für die Zeit der Durchtrennung der Haut durch die Injektion bzw. Punktion anhalten soll, ist bei präoperativer Hautantiseptik eine remanente Wirkung auf die residente Hautflora für die Dauer von der Abdeckung des Operationsfelds bis zum Wundverschluss erwünscht. Alkohole haben zwar eine hohe Sofortwirkung, sind aber nicht remanent wirksam. Durch Zusatz eines remanent wirkenden Antiseptikums zur alkoholischen Formulierung ist jedoch eine remanente Wirkung erreichbar, wodurch die Wirkung auf die Hautflora zeitabhän- 
gig mehr oder weniger ausgeprägt erhöht wird. Als antiseptische Zusätze werden derzeit v. a. Chx, PVP-Iod und Octenidindihydrochlorid (Oct) eingesetzt. Relevant für die Auswahl des Hautantiseptikums sind Studien, die zur präoperativen Hautantiseptik mit der SSI-Rate als Endpunkt durchgeführt wurden. Hierbei wurden Hautantiseptika mit unterschiedlicher Wirkstoffbasis verglichen (- Tab. 1).

Diese Studien belegen, dass wässrige PVP-Iod-Lösung sowohl wässriger als auch insbesondere alkoholbasierter ChxLösung an Wirksamkeit unterlegen ist (- Tab. 1, [54, 62]). Dies lässt sich anhand der SSI-Rate sowie bezüglich der Reduktion der Hautflora nachweisen [11]. Daher ist wässrige PVP-Iod-Lösung nicht mehr als Mittel der Wahl zur Hautantiseptik vor präoperativen Eingriffen anzusehen; hinzu kommt das Risiko resorptiver Nebenwirkungen auf die Schilddrüse [4].

Schwieriger ist die Bewertung der verschiedenen alkoholischen Formulierungen aufgrund unterschiedlicher Studienprotokolle, unterschiedlicher Eingriffe und unterschiedlicher Zusammensetzung der Formulierungen. Durch Zusatz von Chx zu Alkohol wird die Wirksamkeit auf die Hautflora signifikant erhöht, wobei sich die Kombination mit Propan-1ol am wirksamsten erwies [65]. Allerdings steht der Nachweis der höheren Effektivität für die Prävention von SSI durch den Zusatz von Chx zur alkoholischen Formulierung mittels randomisierter kontrollierter Studien (RCT) bisher aus. Ebenso ist alkoholbasierte Chx-Lösung in der Wirksamkeit auf die residente Hautflora wässriger Chx-Lösung überlegen [29]. Bisher fehlt eine RCT, in der die Kombination von $2 \% \mathrm{Chx}$ mit $70 \%$ Propan-2-ol gegen die Kombination von PVP-Iod mit $70 \%$ Propan-2-ol bei identischer Einwirkungszeit und ohne vorherige Waschung der Haut im Operationsareal verglichen wird. Nur in der Studie von Berry et al. [9] wurden alkoholische Formulierungen mit Chx bzw. PVP-Iod-Zusatz verglichen. Allerdings ist die Verblindung unklar und es sind weder der Alkohol noch die Konzentration von PVP-Iod angegeben. Als Ergebnis einer Metanalyse wird anhand lediglich einer Studie für aseptische Eingrif-

Trauma Berufskrankh 2015 • [Suppl 2]: 17:322-329 DOI 10.1007/s10039-015-0026-4

(c) Springer-Verlag Berlin Heidelberg 2015

\section{A. Kramer · C.-D. Heidecke}

\section{Präoperative Hautantiseptik und Hautschutz}

\section{Zusammenfassung}

Hintergrund. Die präoperative Hautantiseptik ist ein essenzieller Bestandteil der Multibarrierenstrategie zur Prävention postoperativer Wundinfektionen (SSI).

Methoden. Für die Hautantiseptik sind alkoholbasierte Formulierungen aufgrund ihrer raschen Wirkung und hohen Wirksamkeit Wirkstoff der ersten Wahl. Durch Zusatz remanent wirksamer Antiseptika (Chlorhexidin Octenidin) wird die Wirkung auf die Hautflora zeitabhängig mehr oder weniger ausgeprägt erhöht. Obwohl der Nachweis der höheren Effektivität alkoholischer Formulierungen mit remanentem Zusatz für die Prävention von SSI mittels randomisierter klinischer Studien bisher aussteht, ist es zugleich unter Berücksichtigung der höheren Wirksamkeit derartiger Formulierungen beim Legen und der Pflege von zentralen Venenkathetern sinnvoll, zur präoperativen Hautantiseptik insbesondere bei lang dauernden Eingriffen Alkohole mit Zusatz eines remanenten Antiseptikums anzuwenden. Entscheidend für die Wirkungsentfaltung ist das mecha- nisch assistierte Einreiben des Antiseptikums für 30 s in die Haut mit anschließender Benetzung der Haut für die Dauer der deklarierten Einwirkungszeit. Wässrige PVP-lod-Lösungen sind aufgrund ihrer signifikant geringeren Wirksamkeit zur präoperativen Hautantiseptik ungeeignet. Durch Hautschutzcremes wird die Barrierefunktion der Haut verbessert und das Risiko einer Irritationsdermatose herabgesetzt, während Hautpflegecremes dem Erhalt bzw. der Verbesserung der Hautfunktionen dienen.

Schlussfolgerung. Bereits bei kurzzeitiger Anwendung von Hautschutz und Hautpflege für 8 bzw. 9 Tage war bei chirurgisch tätigen Ärzten eine Verbesserung des Hautzustands nachweisbar, ohne dass die Wirksamkeit der Händedesinfektion dadurch beeinträchtigt wurde.

\section{Schlüsselwörter}

Wundinfektionen - Antiinfektiva · Alkohole . Chlorhexidin · Octenidin

\section{Preoperative skin antisepsis and skin protection}

\section{Abstract}

Background. Preoperative skin antisepsis is an essential component of multibarrier strategies for the prevention of surgical site infections (SSI).

Methods. Alcohol-based formulations are the preferred agents of choice for achieving preoperative skin antisepsis due to the rapid action and high efficacy. Through the addition of residually acting antiseptics (e.g. chlorhexidine and octenidine), the time-dependent effect on skin flora is ecognizably increased to a greater or lesser extent. Although evidence of greater effectiveness using such alcohol-based formulations together with residually acting antiseptics for the prevention of SSIs from randomized controlled trials is still lacking, their selection is useful particularly during long surgical procedures as well as in the maintenance of central venous catheters. Important for effective de- ployment is a mechanically assisted rubbing of the antiseptic onto the skin for $30 \mathrm{~s}$, with a subsequent wetting of the skin for the duration of the declared exposure time. Aqueous PVP-iodine solutions are inappropriate due to the significantly lower efficacy for preoperative skin antisepsis. The barrier function of the skin is improved through the use of skin protection creams and the risk of irritation dermatitis is reduced, while skincare creams help to preserve and improve the skin's functions. Conclusion. An improvement in skin condition among surgeons was detectable even after short-term use of skin protection and skincare for 8 or 9 days. The effectiveness of hand disinfection was not affected.

\section{Keywords}

Wound infections - Anti-infective agents .

Alcohols · Chlorhexidine · Octenidine fe abgeleitet, dass die präoperative Antiseptik mit 0,5\% Chx in Ethanol mit signifikant geringeren SSI-Raten assoziiert ist als mit ethanolbasierten PVP-Iod-Zubereitungen; allerdings sind Bias nicht auszuschließen [16]. Eine weitere Metana- lyse kommt unabhängig vom Eingriff zu der Schlussfolgerung, dass die Chx-basierte Hautantiseptik der mit PVP-Iod überlegen ist, wobei sowohl wässrige als auch Propan-2-ol-basierte Zubereitungen verglichen wurden, was nicht akzeptabel 
Tab. 2 Einfluss von Hautschutz und Hautpflege auf den Hautzustand

\begin{tabular}{llll}
\hline Parameter & Messreihe $\mathbf{1}$ & Messreihe 2 & Messreihe 3 \\
\hline TEWL & 27,5 & $24,4^{*}$ & 28,2 \\
\hline Lipidgehalt & 1,7 & 1,3 & $5,3^{*}$ \\
\hline AOP & 3,5 & 3,7 & $4,0^{*}$
\end{tabular}

TEWL transepidermaler Wasserverlust, $A O P$ antioxidatives Potenzial.

${ }^{*} p<0,05$.

ist [49]. Signifikant war der Unterschied beim Vergleich zweier alkoholbasierter Zubereitungen nur in der schon zitierten Studie von Berry et al. [9], deren Aussagekraft jedoch eingeschränkt ist.

Insgesamt kann festgestellt werden, dass es sinnvoll ist, zur präoperativen Hautantiseptik, insbesondere bei lang dauernden Eingriffen, Alkohole mit $\mathrm{Zu}$ satz eines remanenten Antiseptikums anzuwenden. Dies gründet sich auf die in - Tab. 1 zusammengefassten Befunde und die Überlegenheit alkoholischer Formulierungen mit Zusatz von Chx im Vergleich zu Alkoholen ohne Zusatz von Chx in Bezug auf die Reduktion der residenten Hautflora sowie in Bezug auf die höhere Wirksamkeit bei dem Legen und der Pflege von zentralen Venenkathetern (ZVK). Durch die Anwendung von Alkoholen mit Zusatz von Chx bzw. Oct wird beim ZVK nicht nur die Rekolonisation der Katheterspitze signifikant verzögert $[13,14,59]$, sondern auch die Inzidenz ZVK-assoziierter Blutstrominfektionen reduziert [31].

Wird Chx mittels Textil oder Applikator aufgetragen, ist die Wirksamkeit höher als bei üblicher Benetzung [18, 80]. Allerdings wird die Wirksamkeit der sog. assistierten Anwendung mit Applikator auch durch mechanisches Einreiben mittels Kornzange und Tupfer erreicht [80]. Daraus ist abzuleiten, dass die präoperative Hautantiseptik zweistufig durchgeführt werden sollte, beginnend mit mechanisch intensivierter Auftragung für $30 \mathrm{~s}$ und nachfolgender Benetzung für die Gesamtdauer der Antiseptik mit der vom Hersteller empfohlenen Einwirkungszeit.

Da in den USA und vielen europäischen Staaten bisher nur Chx-basierte Antiseptika eingesetzt werden, wurden fast alle Studien mit diesem Wirkstoff durchgeführt. Octenidin ist aus folgenden Gründen als eine mindestens gleichwertige Alternative zu Chx anzusehen:
- Octenidin übertrifft in vitro Chx an Wirksamkeit [37].

- Es ist analog zu Chx remanent wirksam bei besserer Biokompatibilität [60].

- In Kombination mit Alkoholen ist Oct vergleichbar effektiv wie Chx in Bezug auf die Reduktion der Hautflora um die Insertion des ZVK $[13,14]$.

- Durch Oct war keine Resistenzentwicklung induzierbar [1, 32]. Dagegen wurde durch Chx eine Resistenzentwicklung sowohl adaptiv als auch R-Plasmid-vermittelt mit Kreuzresistenz gegen Antibiotika beschrieben $[21,74]$.

- Inzwischen wurden v. a. Staphylokokkenisolate von Patienten mit verminderter In-vitro-Empfindlichkeit gegen Chx beschrieben [30, 56, 57]; der Resistenzmechanismus beruht auf plasmidkodierten Effluxpumpen [21].

- Ebenso wurden für Oct im Unterschied zu Chx keine allergischen Kontaktekzeme [71] - wenn sie auch selten auftreten - und IgE-vermittelten anaphylaktischen Reaktionen beschrieben $[32,64]$.

- Dem Bundesinstitut für Arzneimittel und Medizinprodukte (BfArM) lagen bis 2013147 Berichte aus Deutschland über anaphylaktische Reaktionen im Zusammenhang mit der Anwendung von Chx vor [10].

- Schließlich stellt die in der Mundhöhle nachgewiesene Freisetzung der im Chx-Molekül vorhandenen 4-Chloranilin-Gruppe, die als Kanzerogen eingestuft ist, [6] eine weitere Einschränkung dar.

\section{Zulassung}

Hautantiseptika sind in Deutschland als Arzneimittel registriert. Ihre Wirksamkeit muss durch Gutachten mit den für die Zulassung erforderlichen Prüfmethoden des VAH nachgewiesen werden. Als Voraussetzung für die Aufnahme in die Desinfektionsmittelliste des VAH müssen Hautantiseptika in der deklarierten Einwirkungszeit die gleiche oder eine signifikant höhere Wirksamkeit besitzen als das Referenzverfahren mit Propan-2-ol 70 Vol.\% [23].

Die erforderliche und zugleich gelistete Einwirkungszeit der präoperativen Antiseptik ist allerdings weder experimentell noch epidemiologisch gesichert. Da der Talgdrüsenanteil regional unterschiedlich ist und der Fettgehalt der Haut individuellen Schwankungen unterliegt, ist man auf der sicheren Seite, wenn auch auf talgdrüsenarmen Arealen die präoperative Hautantiseptik mit der Einwirkungszeit für talgdrüsenreiche Haut zugrunde gelegt wird, zumal mit alkoholbasierten Präparaten bei Auftragung ohne anschließendes mechanisches Einreiben nur eine Reduktion auf der Hautoberfläche um etwa $1,2 \log _{10}$ erreicht wird [80] und der Alkohol nicht in die Haarfollikel eindringt $[78,79]$.

\section{Durchführung}

Die Hautantiseptik wird im Operationsraum durchgeführt. Die Haut muss während der vom Hersteller deklarierten Einwirkzeit satt benetzt und feucht gehalten werden. Hierbei ist darauf zu achten, dass der Patient nicht in Flüssigkeitsansammlungen des Antiseptikums zu liegen kommt, da dies zu Hautschädigung (Nekrosen) oder Komplikationen durch Kriechströme beim Kauterisieren führen kann. Nach erfolgter Antiseptik wird die Umgebung des Operationsgebiets steril abgedeckt [42]. Durch Freigabe des Hautantiseptikums mittels Applikator auf ein Schwämmchen werden eine Standardisierung und zugleich höhere Effektivität erreicht. Alternativ ist das Vorgehen mit Tupfer und Kornzange mit gleicher Effektivität standardisierbar [80].

Obwohl Alkohole bei Körperwärme flüchtig sind und rasch verdunsten, ist bei großflächiger Applikation die Brandgefahr zu beachten, da Alkohol-Luft-Gemische entzündbar sind. Ein besonderes Risiko besteht, wenn in der Nähe Zündquellen vorhanden sind (z. B. Elektro- 


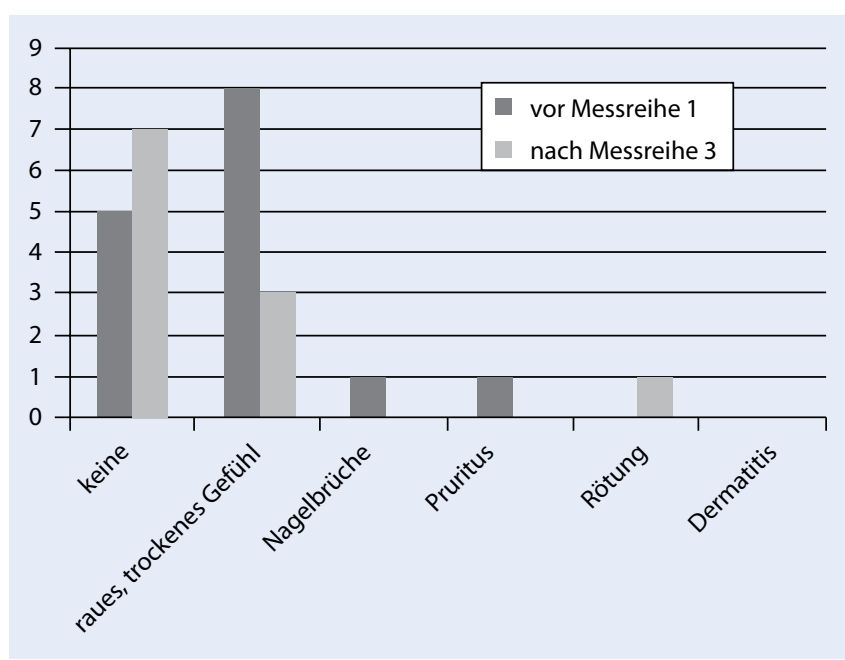

Abb. $1<$ Einfluss von Hautschutz und Hautpflege auf Hautveränderungen kauter, offene Flamme; [38]). Ferner ist zu berücksichtigen, dass alle kurzkettigen Alkohole dermal resorbiert werden $[5,39]$. Deshalb empfiehlt sich bei besonders empfindlichen Patienten (Frühund Neugeborene, Patienten mit Leberschädigung) die Anwendung ethanolhaltiger Antiseptika, da Ethanol im Vergleich zu Propan-1-ol und Propan-2-ol toxikologisch unkritisch ist. Die Anwendung wässriger Präparate auf Oct-Basis kann bei Anwendung an Haut-SchleimhautÜbergängen oder bei unreifen Neonaten [42] notwendig sein.

\section{Hautschutz und Hautpflege}

\section{Bedeutung}

Berufsbedingte Hauterkrankungen stehen seit vielen Jahren an der Spitze der Berufskrankheiten [19]. Ein Grund hierfür dürfte sein, dass die Hände zu viel gewaschen werden, anstatt alkoholische Händedesinfektionsmittel zu benutzen. Zum anderen werden Hautschutz- und Hautpflegemittel aus Unkenntnis zu wenig oder auch falsch angewendet [20, 72]. Sowohl bei Pflegepersonal als auch bei Ärzten ergaben sich große Wissensdefizite in Hinblick auf Hautschutz und Hautpflege $[24,25]$. Deshalb ist die Wissensvermittlung in Verbindung mit der Etablierung eines Hautschutzplans wichtig und leistet einen Beitrag zur Verbesserung beruflich irritierter Haut $[45,51,85]$.

Hautschutzpräparate schützen vor Irritation [7, 22, 48] und werden vor der Arbeit und ggf. zusätzlich in den Arbeits- pausen aufgetragen [8]. Hautpflegepräparate unterstützen die Regeneration der Haut und damit ihre Barrierefunktion [75]. Bei sichtbarer Verschmutzung der Hände soll vor dem Auftragen von Hautschutz- oder -pflegepräparaten die Haut gereinigt werden, um dem Eindringen von auf der Haut verbliebenen Substanzen mit hautreizenden Eigenschaften entgegenzuwirken [41].

Auch wenn Hautschutz und Hautpflege vorrangig dem Arbeitsschutz dienen, ist eine glatte gepflegte Haut Voraussetzung für eine effektive Händedesinfektion [55], da bereits kleinste Risse bzw. Mikrotraumen zum Erregerreservoir werden können [46].

\section{Umsetzung in die Praxis}

Bei Gefährdung der Haut durch Arbeiten im feuchten Milieu - dazu gehört auch das Tragen flüssigkeitsdichter Handschuhe $>2 \mathrm{~h}-$ muss der Arbeitgeber persönliche Schutzausrüstung bereitstellen, eine Betriebsanweisung und einen Hautschutzplan erstellen, die Möglichkeit zur Reduzierung der Feuchtigkeitsexposition einschließlich Ersatzstoffprüfung überprüfen und die arbeitsmedizinische Vorsorge und Überwachung gewährleisten [77].

$\mathrm{Zu}$ Dienstbeginn sollten die Hände kurz gewaschen und gründlich getrocknet werden. Danach ist eine Hautschutzcreme gleichmäßig über die Hände einschließlich Fingerzwischenräume zu verteilen. Nach größeren Arbeitsunterbrechungen, z. B. Mittagspause, empfiehlt sich die erneute Anwendung der Hautschutzcreme. Zwischenzeitlich sollte die Hautpflegecreme bei individuellem Bedürfnis, z. B. Gefühl trockener Hände, und ebenso am Arbeitsende benutzt werden. Beim Umgang mit irritativen Noxen (z. B. Flächendesinfektion, Instrumentenaufbereitung) sind Schutzhandschuhe (keine Untersuchungshandschuhe) anzulegen. Bei beginnenden Hautschäden ist der betriebsärztliche Dienst oder ein Hautarzt zu konsultieren.

Ob ein zeitlicher Abstand zwischen der Anwendung von Hautschutz- und Hautpflegepräparaten zur Händedesinfektion eingehalten werden muss, ist bisher nicht ausreichend untersucht. In praxisfremden Modellen wurde eine Wirkungsverminderung für alkoholische Händedesinfektionsmittel durch Pflegeprodukte [69] nachgewiesen. Die Association for Professionals in Infection Control (APIC) empfiehlt deshalb, die Kompatibilität von Pflegeprodukt und Händedesinfektionsmittel bereits bei der Produktauswahl zu berücksichtigen, gibt jedoch keine Hinweise zu einem Verfahren der Kompatibilitätsprüfung [47].

Nach Anwendung von Hautschutz und Durchführung einer hygienischen Händedesinfektion nach 5 min bzw. 60 min wurde keine Wirkungsbeeinflussung festgestellt [24]. Nach Anwendung von Hautschutz und Hautpflege (je 3-mal/Tag für 9 Tage) bei einem chirurgischen Team wurde der Hautzustand signifikant verbessert, ohne dass auch hier die Wirksamkeit der Händedesinfektion beeinträchtigt wurde [25].

In einer noch unveröffentlichten prospektiven Studie zum dermatologischen Nutzen von Hautschutz in der Chirurgie wurden nach täglicher Erhebung der Vorwerte innerhalb von 2 Wochen (Messreihe 1) zwei weitere Messreihen für je 1 Woche bei 11 Probanden durchgeführt [28]:

Messreihe 2. Anwendung des Hautschutzprodukts Praecutan ${ }^{\circledR}$ TwinProtect sensitiv und des Hautpflegeprodukts Praecutan ${ }^{\oplus}$ Creme sensitive (beide Evonik Industries AG, Krefeld).

Messreihe 3. Ersatz von Praecutan ${ }^{\circledR}$ Creme sensitive durch das Hautpflegepro- 
dukt Apomix (PKH GmbH, Halle) wegen unangenehmen Auftrageverhaltens (krümelige Konsistenz, negative Compliance) und Auftretens von verschlechtertem Hautbild mit Desquamation und Rubor bei einem Probanden. In Messreihe 2 musste wegen einer Noroviruserkrankung das Händedesinfektionsmittel Sterillium ${ }^{\circ}$ classic pure gegen das viruzid wirksame Händedesinfektionsmittel Manorapid ${ }^{\circledR}$ Synergy ausgewechselt werden, das erfahrungsgemäß deutlich schlechter von der Haut toleriert wird.

Die Hautpflege wurde 1- bis 5-mal/ Tag durchgeführt, der Hautschutz wurde von 10 Probanden ebenfalls 1- bis 5-mal/ Tag und von 1 Probanden 5- bis 10-mal/ Tag aufgetragen. Bestimmt wurden der transepidermale Wasserverlust (TEWL), der Hautlipidgehalt und das antioxidative Potenzial (AOP) der Haut.

Trotz des Wechsels auf ein viruzides Händedesinfektionsmittel in Messreihe 2 verbesserten sich der Hautlipidgehalt und das AOP. Lediglich der TEWL wurde tendenziell reduziert ( $\bullet$ Tab. 2). Die subjektive Einschätzung von 0 „keinerlei Hautveränderungen“ bis 10 „extrem angegriffen" ergab im Vergleich der Vorwerte von Messreihe 1 und nach Messreihe 3 trotz Anwendung des viruziden Händedesinfektionsmittels keinen Unterschied und subjektive Hautveränderungen besserten sich (• Abb. 1).

Damit konnte trotz der kurzen Studiendauer von 2 Wochen ein günstiger Einfluss von Hautschutz und Hautpflege in einem chirurgischen Team nachgewiesen werden [28].

Da trotz dieser ersten orientierenden Untersuchungen nicht auszuschlieBen ist, dass einige Hautpflegemittel die Wirkung alkoholischer Händedesinfektionsmittel beeinträchtigen können, ist deren Anwendung - sofern ihr Einfluss auf die Wirksamkeit der Händedesinfektion nicht untersucht ist - am günstigsten in Arbeitspausen und zusätzlich nach Arbeitsschluss vorzunehmen.

Eine weitere Möglichkeit zum Schutz der Haut ist das Tragen von Baumwollunterziehhandschuhen unter dem Schutzhandschuh, wodurch die Schweißansammlung reduziert und damit die subjektiv empfundene Hautverträglichkeit verbessert wird [33].

\section{Korrespondenzadresse}

Prof. Dr. A. Kramer

Institut für Hygiene und Umweltmedizin

Universitätsmedizin Greifswald

Walter-Rathenau-Straße 49 A, 17475 Greifswald

kramer@uni-greifswald.de

\section{Einhaltung ethischer Richtlinien}

Interessenkonflikt. A. Kramer und C.-D. Heidecke geben an, dass kein Interessenkonflikt besteht.

Dieser Beitrag beinhaltet keine Studien an Tieren. Die prospektive Studie zum Nachweis des dermatologischen Nutzens von Hautschutz- und Hautpflegecreme bei einem chirurgischen Team wurde von der Ethikkommission der Universität Greifswald befürwortet (Interne Reg.Nr. BB 134/13).

The supplement containing this article is not sponsored by industry.

\section{Literatur}

1. Al-Doori Z, Goroncy-Bermes P, Gemmell CG, Morrison D (2007) Low-level exposure of MRSA to octenidine dihydrochloride does not select for resistance. J Antimicrob Chemother 59:1280-1281

2. Alexander J, Aerni S, Plettner J (1985) Development of a safe and effective one-minute preoperative skin preparation. Arch Surg 120(12):13571361

3. Amer-Alshiek J, Alshiek T, Almog B, Lessing JB, Satel A, Many A, Levin I (2013) Can we reduce the surgical site infection rate in cesarean sections using a chlorhexidine-based antisepsis protocol? J Maternal-Fetal Neonat 26(17):1749-1752

4. Below H, Brauer V, Kramer A (2007) lodresorption bei antiseptischer Anwendung von lodophoren und Schlussfolgerungen zur Risikobewertung. GMS Krankenhaushyg Interdiszip 2(2):Doc30

5. Below H, Partecke I, Hübner NO, Bieber N, Nicolai T, Usche A, Assadian O, Below E, Kampf G, Parzefall W, Heidecke CD, Zuba D, Bessonneau V, Kohlmann T, Kramer A (2012) Dermal and pulmonary absorption of propan-1-ol and propan-2-ol from hand rubs. Am J Infect Control 40(3):250-257

6. Below H, Assadian O, Hildebrandt U et al Prevalence of chlorhexidine digluconate, 4-chloraniline, and 4-chlornitrobenzene in saliva after pre- and postoperative mouth rinse with $0.2 \%$ chlorhexidine digluconate. In preparation

7. Berndt U, Wigger-Alberti W, Gabard B, Elsner $P$ (2001) Vergleich einer Hautschutzcreme und ihrer Grundlage bezüglich Wirksamkeit gegen das berufsbedingte irritative Handekzem bei Krankenschwestern/Eine Anwendungsuntersuchung. Derm Beruf Umwelt 49(1):77-80

8. Berndt U, Gabard B, Schliemann-Willers S, Wigger-Alberti W, Zitterbart D, Elsner P (2002) Integrated skin protection from workplace irritants: a new model for efficacy assessment. Exogenous Dermatol 1(1):45-48

9. Berry A, Watt B, Goldacre M, Thomson J, McNair T (1982) A comparison of the use of povidone-iodine and chlorhexidine in the prophylaxis. J Hosp Inf 3(1):55-63
10. Bundesinstitut für Arzneimittel und Medizinprodukte (BfArM) (2013) Chlorhexidin: Anaphylaktische Reaktionen. http://www.bfarm.de/DE/Pharmakovigilanz/risikoinfo/2013/RI chlorhexidin. html. Zugegriffen: 27. Sept. 2013

11. Culligan PJ, Kubik K, Murphy M, Blackwell L, Snyder $\mathrm{J}$ (2005) A randomised trial that compared povidoneiodine and chlorhexidine as antiseptics for vaginal hysterectomy. Am J Obstet Gynecol 192:422425

12. Darouiche RO, Wall MJ, Itani KM, Otterson MF, Webb AL, Carrick MM, Miller HJ, Awad SS, Crosby CT, Mosier MC, Alsharif A, Berger DH (2010) Chlorhexidine-alcohol versus povidone-iodine for surgical-site antisepsis. N Engl J Med 362(1):18-26

13. Dettenkofer M, Jonas D, Wiechmann C, Rossner R, Frank U, Zentner J, Daschner FD (2002) Effect of skin disinfection with octenidine dihydrochloride on insertion site colonization of intravascular catheters. Infection 30(5):282-285

14. Dettenkofer M, Wilson C, Gratwohl A, Schmoor C, Bertz H, Frei R, Heim D, Luft D, Schulz S, Widmer AF (2010) Skin disinfection with octenidine dihydrochloride for central venous catheter site care: a double-blind, randomized, controlled trial. Clin Microbiol Infect 16(6):600-606

15. Dohmen PM, Gabbieri D, Weymann A, Linneweber J, Geyer T, Konertz W (2011) A retrospective nonrandomized study on the impact of INTEGUSEAL, a preoperative microbial skin sealant, on the rate of surgical site infections after cardiac surgery. Int J Infect Dis 15(6):395-400

16. Dumville JC, McFarlane E, Edwards P, Lipp A, Holmes A (2013) Preoperative skin antiseptics for preventing surgical wound infections after clean surgery. Cochrane Database Syst Rev 3:CD003949

17. Eckardstein von AS, Lim CH, Dohmen PM, PegoFernandes PM, Cooper WA, Oslund SG, Kelley EL (2011) A randomized trial of a skin sealant to reduce the risk of incision contamination in cardiac surgery. Ann Thorac Surg 92(2):632-637

18. Edmiston CE, Seabrook GR, Johnson CP, Paulson DS, Beausoleil CM (2007) Comparative of a new and innovative $2 \%$ chlorhexidine gluconate-impregnated cloth with $4 \%$ chlorhexidine gluconate as topical antiseptic for preparation of the skin prior to surgery. Am J Infect Control 35(2):89-96

19. Fartasch $M$, Diepgen $T L$, Drexler $H$, Elsner $P$, Fluhr JW, John SW, Kresken J, Wigger-Alberti W (2009) Berufs-dermatologie - Berufliche Hautmittel (ICD10: L23, L24) S 1-Leitlinie der Arbeitsgemeinschaft für Berufs und Umweltdermatologie (ABD) in der Deutschen Dermatologischen Gesellschaft (DDG). Arbeitsmedizin Sozialmedizin Umweltmedizin 44(2):53

20. Forrester BG, Roth VS (1998) Hand dermatitis in intensive care units. J Occup Environm Med 40(10):881-885

21. Fritz SA, Hogana PG, Camins BC, Ainswortha AJ, Patricka C, Martina MS, Kraussc MJ, Rodrigueza M, Burnhama CAD (2013) Mupirocin and chlorhexidine resistance in Staphylococcus aureus in patients with community-onset skin and soft tissue infections. Antimicrob Agents Chemother 57(1):559-568

22. Frosch PJ, Kurte A (1994) Efficacy of skin barrier creams (IV). The repetitive irritation test (RIT) with a set of 4 standard irritants. Contact Derm 31(3):161-168

23. Gebel J, Werner HP, Kirsch-Altena A, Bansemir K (2001) Standardmethoden der DGHM zur Prüfung chemischer Desinfektionsverfahren. mhp, Wiesbaden 
24. Große-Schütte K, Assadian O, Hübner NO, Löffler $\mathrm{H}$, Kramer A (2011) Practices of skin care among nurses in medical and surgical intensive care units: results of a self-administered questionnaire. GMS Krankenhaushygiene Interdisziplinar 6(1):Doc08

25. Harnoss JC, Brune L, Ansorg J, Heidecke CD, Assadian O, Kramer A (2014) Practice of skin protection and skin care among German surgeons and influence on the efficacy of surgical hand disinfection and surgical glove perforation. BMC Infect Dis 14(1):315

26. Heeg P, Christiansen B (1993) Hautantiseptik. In: Kramer A, Gröschel D, Heeg P, Hingst V, Lippert H, Rotter M, Weuffen W (Hrsg) Klinische Antiseptik. Springer, Berlin Heidelberg New York, S 105-119

27. Heeg P, Kramer A, Pitten FA, Rudolph P (1998) Hautantiseptik aus prophylaktischer Indikation. Hygiene in Krankenhaus und Praxis. Ecomed Landsberg, S 1-7

28. Henschel W (2014) Prospektive Pilotstudie zum dermatologischen Nutzen der Einführung von Hautschutz- und Hautpflegecreme in ein chirurgisches Team. Diss Universitätsmedizin Greifswald

29. Hibbard JS, Mulberry GK, Brady AR (2002) A clinical study comparing the skin antisepsis and safety of Chloraprep, $70 \%$ isopropyl alcohol, and $2 \%$ aqueous chlorhexidine. J Infusion Nurs 25(4):244249

30. Ho CM, Li CY, Ho MW, Lin CY, Liu SH, Lu JJ (2012) High rate of qacA and qacB positive methicillin resistant Staphylococcus aureus isolates from chlorhexidine impregnated catheter related bloodstream infections. Antimicrob Agents Chemother 56:5693-5697

31. Huang EY, Chen C, Abdullah F, Aspelund G, Barnhart DC, Calkins CM, Cowles RA, Downard CD, Goldin AB, Lee SL, Peter SDS, Marjorie J, Arca MJ (2011) For the 2011 American Pediatric Surgical Association Outcomes and Clinical Trials Committee. Strategies for the prevention of central venous catheter infections: an American Pediatric Surgical Association Outcomes and Clinical Trials Committee systematic review. J Ped Surg 46:2000-2011

32. Hübner NO, Siebert J, Kramer A (2010) Octenidine dihydrochloride, a modern antiseptic for skin, mucous membranes and wounds. Skin Pharmacol Physiol 23(5):244-258

33. Hübner NO, Rubbert K, Pohrt U, Heidecke CD, Partecke LI, Kramer A (2014) Einsatz wiederaufbereitbarer textiler Unterziehhandschuhe für medizinische Tätigkeiten: eine Machbarkeitsstudie. Zbl Chir 139:1-6

34. Iyer A, Gilfillan I, Thakur S, Sharma S (2011) Reduction of surgical site infection using a microbial sealant: a randomized trial. J Thorax Cardiovasc Surg 142:438-442

35. Kampf G, Kramer A (2004) Epidemiologic background of hand hygiene and evaluation of the most important agents for scrubs and rubs. Clin in Microbiol Rev 17(4):863-893

36. Kirkland KB, Briggs JP, Trivette SL, Wilkinson WE, Sexton DJ (1999) The impact of surgical site infections in the 1990s: attributable mortality, excess length of hospitalization, and extra costs. Infect Control Hosp Epidemiol 20:725-730

37. Koburger T, Hübner NO, Braun M, Siebert J, Kramer A (2010) Standardized comparison of antiseptic efficacy of triclosan, PVP-iodine, octenidine dihydrochloride, polyhexanide and chlorhexidine digluconate. J Antimicrob Chemother 65(8):1712-1719

38. Kramer A, Kampf G (2007) Hand rub-associated fire incidents during 25,038 hospital-years in Germany. Infect Control Hosp Epidemiol 28(6):745746
39. Kramer A, Below H, Bieber N, Kampf G, Toma CD, Hübner NO, Assadian O (2007) Quantity of ethanol absorption after excessive hand disinfection using three commercially available hand rubs is minimal and below toxic levels for humans. BMC Infect Dis 7(1):117

40. Kramer A, Assadian O, Gruber B, Lademann J (2008) Prävention von postoperativen Wundinfektionen, Teil 1: Präoperative Maßnahmen - Einfluss der Haarentfernung. Hyg Med 33(10):402-407

41. Kresken J. Klotz A (2003) Occupational skin-protection products - a review. Int Arch Occup Environm Health 76(5):355-358

42. KRINKO; Oldhafer K, Jürs U, Kramer A, Martius J, Weist K, Mielke M) (2007) Prävention postoperativer Infektionen im Operationsgebiet. Bgbl Gesundheitsforsch Gesundheitsschutz 50:377-393

43. KRINKO; Simon A, Christoph J, Geffers C, Hentschel J, Jürs U, Kramer A, Laux R, Müller A, Wendt C) (2007).Empfehlung zur Prävention nosokomialer Infektionen bei neonatologischen Intensivpflegepatienten mit einem Geburtsgewicht unter $1500 \mathrm{~g}$. Bgbl Gesundheitsforsch Gesundheitsschutz 50:1265-1303

44. KRINKO (2010) Die Kategorien in der Richtlinie für Krankenhaushygiene und Infektionsprävention Aktualisierung der Definitionen. Bgbl 53:754-756

45. Kütting B, Drexler H (2008) Der dreistufige Hautschutzplan. Dtsch Med Wschr 133(05):201-205

46. Lammers T (1978) Zur Prüfung der Händedesinfektion. Hyg Med 3:316-318

47. Larson EL (1995) APIC guidelines for handwashing and hand antisepsis in health care settings. Am J Inf Contr 23(4):251-269

48. Larson E, Anderson JK, Baxendale L, Bobo L (1993) Effects of a protective foam on scrubbing and gloving. Am J Inf Contr 21(6):297-301

49. Lee I, Agarwal RK, Lee BY, Fishman NO, Umscheid CA (2010) Systematic review and cost analysis comparing use of chlorhexidine with use of iodine for preoperative skin antisepsis to prevent surgical site infection. Inf Contr Hosp Epidemiol 31(12):1219-1229

50. Levin I, Alshiek JA, Avni A, Lessing JB, Satel A, Almog $B$ (2011) Chlorhexidine and alcohol versus povidone-iodine for antisepsis in gynecological surgery. JWomen's Health 20(3):321-324

51. Löffler H, Dickel H, Bruckner T, Effendy I, Happle $R$ (2002) Skin changes in geriatric nurses prior to training heralding a particular risk of hand dermatitis. Eur J Dermatol 12(5):452-454

52. Maier S, Kramer A, Heidecke CD (2010) Vermeidung und Therapie postoperativer Infektionen. Allgemein Viszeralchir up2date 6:325-344

53. Maier S, Heidecke CD, Kramer A (2014) Prävention von Surgical Site Infections (SSI). Zbl Chir 139(2):139-144

54. Maiwald M, Chan ESY (2012) The forgotten role of alcohol: a systematic review and meta-analysis of the clinical efficacy and perceived role of chlorhexidine in skin antisepsis. PLoS ONE 7(9):e44277

55. Mäkelä $P$ (1993) Gesunde Haut als Voraussetzung für eine effektive Händedesinfektion. In: Kramer A Gröschel D, Heeg P, Hingst V, Lippert H, Rotter M, Weuffen W (Hrsg) Klinische Antiseptik. Springer, Berlin Heidelberg New York, S 97-103

56. McNeil JC, Hulten KG, Kaplan SL, Mahoney DH, Mason EO (2013a) Staphylococcus aureus infections in pediatric oncology patients: high rates of antimicrobial resistance, antiseptic tolerance and complications. J Pediatr Infect Dis 32:124-128
57. McNeil J, Ligon J, Hulten K, Dreyer J, JS H, Mason E, Kaplan S (2013a) Staphylococcus aureus infections in children with congenital heart disease. J Pediatr Infect Dis 2:337-344

58. Menderes G, Ali NA, Aagaard K, Sangi-Haghpeykar H (2012) Chlorhexidine-alcohol compared with povidone-iodine for surgical-site antisepsis in cesarean deliveries. Obstetr Gynecol 120(5):10371044

59. Mimoz O, Pieroni L, Lawrence C, Edouard A, Costa Y, Samii K, Brun-Buisson C (1996) Prospective, randomized trial of two antiseptic solutions for prevention of central venous or arterial catheter colonization and infection in intensive care unit patients. Crit Care Med 24(11):1818-1823

60. Müller G, Langer J, Siebert J, Kramer A (2014) Residual antimicrobial effect of chlorhexidine digluconate and octenidine dihydrochloride on reconstructed human epidermis. Skin Pharmacol Physiol 27:1-8

61. Nationales Referenzzentrum für die Surveillance von Nosokomialen Infektionen (2012) Deutsche Daten im Rahmen der ersten europäischen Prävalenzerhebung zum Vorkommen nosokomialer Infektionen und zur Antibiotikaanwendung. Epid Bull 26:239-240

62. Noorani A, Rabey N, Walsh SR, Davies RJ (2010) Systematic review and meta-analysis of preoperative antisepsis with chlorhexidine versus povidine-iodine in clean-contaminated surgery. Br J Surg 97:1614-1620

63. Paocharoen V, Mingmalairak C, Apisarnthanarak A (2009) Comparison of surgical wound infection after preoperative skin preparation with $4 \%$ chlorhexidine and povidone iodine: a prospective randomised trial. J Med Assoc Thailand 92(7):898-902

64. Pham NH, Weiner JM, Reisner GS, Baldo BA (2000) Anaphylaxis to chlorhexidine. Case report. Implication of immunoglobulin $\mathrm{E}$ antibodies and identification of an allergenic determinant. Clin Exp Allergy 30:1001-1007

65. Reichel M, Heisig P, Kohlmann T, Kampf G (2009) Alcohols for skin antisepsis at clinically relevant skin sites. Antimicrob Agents Chemother 53(11):4778-4782

66. Rotter ML (2008) Desinfektion der Hände. In: Kramer A, Assadian O (Hrsg) Wallhäußers Praxis der Sterilisation, Desinfektion, Antiseptik und Konservierung. Thieme, Stuttgart, S 146-161

67. Rotter ML, Kramer A (1993) Hygienische Antiseptik. In: Kramer A, Gröschel D, Heeg P, Hingst V, Lippert $\mathrm{H}$, Rotter M, Weuffen W (Hrsg) Klinische Antiseptik. Springer, Berlin Heidelberg New York, S6782

68. Rüden H (1995) Prävalenz nosokomialer Infektionen; Qualitätssicherung in der Krankenhaushygiene. In: Rüden H, Daschner F, Schumacher M (Hrsg) Nosokomiale Infektionen in Deutschland Erfassung und Prävention (NIDEP)-Studie); Teil 1, Schriftenreihe Bundesministerium für Gesundheit Bd 56. Nomos, Baden-Baden

69. Schubert R (1982) Zur Kompatibilität von Hautpflege-Cremes mit Hautdesinfektions-Präparaten. Umweltmedizin 3:56-58

70. Sistla SC, Prabhu G, Sistla S, Sadasivan J (2010) Minimizing wound contamination in a,clean' surgery: comparison of chlorhexidine-ethanol and povidone-iodine. Chemother 56(4):261-267

71. Stingeni L, Lapomarda V, Lisi P (1995) Occupational hand dermatitis in hospital environments. Contact Derm 33:172-176 
72. Stutz NC (2008) Wahrnehmung von Handhygienemaßnahmen durch Pflegepersonal: alkoholische Händedesinfektion versus hygienische Händewaschung-eine Multicenterfragebogenstudie mit anschließender Epikutantestung. Diss Med Fak Univ Marbur

73. Swenson BR, Hedrick TL, Metzger R, Bonatti H, Pruett TL, Sawyer RG (2009) Effects of preoperative skin preparation on postoperative wound infection rates: a prospective study of 3 skin preparation protocols. Inf Contr Hosp Epidemiol 30(10):964971

74. Tattawasart U, Maillard JY, Furr JR, Russel AD (1999) Development of resistance to chlorhexidine diacetate and cetylpyridinium chloride in Pseudomonas stutzeri and changes in antibiotic susceptibility. J Hosp Inf 42:219-229

75. Teichmann A, Sadeyh Pour Soleh H, Schanzer S, Richter H, Schwarz A, Lademann J (2006) Evaluation of the efficacy of skin care products by laser scanning microscopy. Laser Physics Lett 3(10):507509

76. Towfigh S, Cheadle WG, Lowry SF, Malangoni MA, Wilson SE (2008) Significant reduction in incidence of wound contamination by skin flora through use of microbial sealant. Arch Surg 143(9):885-891

77. TRGS 531 (1996) Gefährdung der Haut durch Arbeiten im feuchten Milieu (Feuchtarbeit). Ausschuss für Gefahrstoffe

78. Ulmer M, Patzelt A, Vergou T, Lademann J, Richter H, Kramer A, Müller G, Sterry W, Lange-Asschenfeldt B (2012) In vitro investigation of the follicular penetration of porcine ear skin using a nanoparticle-emulsion containing the antiseptic polihexanide. Laser Phys Lett 9(5):381-386

79. Ulmer M, Patzelt A, Vergou T, Richter H, Müller G, Kramer A, Weltmann KD, Lange-Asschenfeldt B (2013) In vivo investigation of the efficiency of a nanoparticle-emulsion containing polihexanide on the human skin. Eur J Pharm Biopharm 84:325329

80. Ulmer M, Lademann J, Patzelt A, Kramer A, Koburger T, Assadian O, Daeschlein G, Lange- Asschenfeldt B (2014) New strategies for preoperative skin antisepsis. Skin pharmacol 27(6):283-292

81. VAH (2014) Desinfektionsmittel-Liste des VAH. mhp, Wiesbaden

82. Veiga DF, Damasceno CA, Veiga Filho J, Figueiras RG, Vieira RB, Florenzano FH, Juliano Y, Ferreira LM (2008) Povidone iodine versus chlorhexidine in skin antisepsis before elective plastic surgery procedures: a randomized controlled trial. Plast Reconstr Surg 122(5):p170e-171e

83. Waldow T, Szlapka M, Hensel J, Plötze K, Matschke K, Jatzwauk L (2012) Skin sealant InteguSeal ${ }^{\circledR}$ has no impact on prevention of postoperative mediastinitis after cardiac surgery. J Hosp Inf 81(4):278282

84. Webster J, Alghamdi AA (2007) Use of plastic adhesive drapes during surgery for preventing surgical site infection. Cochrane Database Syst Rev 4:pCD006353

85. Weisshaar E, Radulescu M, Bock M, Albrecht U, Zimmermann E, Diepgen TL (2005) Skin protection and skin disease prevention courses for secondary prevention in health care workers: first results after two years of implementation. J Dtsch Dermatol Ges 3(1):33-38

86. Wilson SE (2008) Microbial sealing: a new approach to reducing contamination. J Hosp Inf 70(Suppl 2):11-14 